\title{
The cardiotoxicity of eosinophils
}

\author{
CHRISTOPHER J. F. SPRY \\ M.R.C.P., D.Phil., M.R.C.Path.
}

\author{
POH-CHUN TAI \\ M.Sc., Ph.D.
}

\section{JOHN DAVIES}

M.R.C.P.

Department of Immunology, Royal Postgraduate Medical School, London W12 OHS

\begin{abstract}
Summary
Although an association between high blood eosinophil counts and endomyocardial disease has been known for nearly a hundred years, the reasons for this were not understood. Brockington, Luzzatto and Osunkoya (1970) suggested that eosinophil leucocytes, in susceptible persons, by some unknown mechanisms, cause endomyocardial damage. Evidence to support this possibility has come from three sources: (1) Clinical studies have shown that very high blood eosinophil counts, from any cause, can be associated with endomyocardial disease, and in some patients it has been possible to show that eosinophilia preceded the onset of heart disease. (2) The development of heart disease has been associated with the presence of degranulated eosinophils in the blood and tissues, including damaged endomyocardium, and raised serum levels of eosinophil granule basic proteins have been found in many of these patients. (3) Low concentrations of eosinophil secretion products (which contain these eosinophil granule basic proteins) have been found to injure isolated heart cells in vitro. Studies with purified eosinophil granule basic proteins have shown that cardiac cell damage is the result of a specific toxic effect of eosinophil cationic protein on the plasma membrane and two enzyme complexes (pyruvate dehydrogenase and 2oxoglutarate dehydrogenase) involved in mitochondrial respiration. These results support the suggestion that under certain conditions, eosinophils may damage the heart, leading to endomyocardial disease, and they offer new approaches for the early diagnosis and treatment of endomyocardial disease both in temperate and tropical countries.
\end{abstract}

KEY WORDS: endomyocardial disease, eosinophilia.

\section{Introduction}

There have been clinical reports of an association between high blood eosinophil counts and endomyo- cardial fibrosis for nearly a hundred years, but wide recognition of this was only achieved following the report of two patients studied during life by Löffler (1936). Brockington et al. (1970) described a patient with eosinophilic leukaemia and endomyocardial fibrosis, and were so struck by the association that they suggested that eosinophil leucocytes, in susceptible persons, by some unknown mechanisms, cause endomyocardial damage. Zucker-Franklin (1971) also raised the possibility that eosinophils might damage the heart by analogy with carcinoid induced endocardial lesions. Independently, Yam et al. (1972) noted the frequent association of endocarditis and eosinophilic leukaemia, and felt that this could not be coincidental. They also proposed that there was a cause-effect relationship similar to that of cardiopulmonary lesions in metastatic carcinoid syndrome. In addition, they pointed out that endomyocardial fibrosis had occurred in patients who had a prolonged eosinophilia from many different causes. The possibility that eosinophils might release their toxic constituents and injure the endocardium did not receive wide acceptance, largely because it was held at that time that eosinophils had a modulating or dampening role in inflammation. The later demonstration that eosinophils could kill some parasites in vitro initiated a large amount of subsequent work, which has shown that eosinophils have a marked capacity to injure some organisms and cells (Spry, 1978).

The purpose of this paper is to review recent clinical and experimental studies which have shown that eosinophils have a potent cardiotoxic capacity, which may explain the well-known association between high blood eosinophil counts and endomyocardial fibrosis. Much of this work has been done with purified eosinophils (and their constituents) from patients with the hypereosinophilic syndrome (Spry, 1982; Spry et al., 1983a), over $80 \%$ of whom developed eosinophilic endomyocardial disease (Parrillo et al., 1979; Davies et al., 1983b). It has been 
suggested that a similar pathogenetic mechanism is responsible for the development of tropical endomyocardial fibrosis (Olsen and Spry, 1979), based on the observation that eosinophilic and tropical endomyocardial fibrosis are pathologically indistinguishable (Brockington and Olsen, 1973). If this is so, then there are important implications for therapy and prevention of endomyocardial disease in many patients both in temperate and tropical climates.

\section{Patients and methods}

Clinical features of the patients have been described elsewhere (Spry et al., 1982; Davies et al., 1982). In brief, there were 15 patients with the hypereosinophilic syndrome, 11 of whom had biventricular eosinophilic endomyocardial disease. Five patients presented with heart disease, and 6 others developed cardiac involvement, a mean of 1.3 years after hypereosinophilia was first detected. Nine had mitral valve disease and 6 had tricuspid regurgitation. Cardiac histology showed acute necrotic lesions in 4 patients and late fibrotic lesions in 5 . The mean blood eosinophil count at presentation was $20.1 \times 10^{9}$ /litre. A mean of $3.7 \times 10^{9} /$ litre degranulated blood eosinophils (Spry and Tai, 1976) were found in the blood of 10 patients.

Measurements were made of the capacity of patients' blood eosinophils to injure isolated rat heart cells in vitro, as described previously (Tai et al., 1982). Rat heart cells were isolated by collagenase and hyaluronidase digestion, and incubated with supernatants from purified eosinophils which had been stimulated with zymosan- $\mathrm{C}_{3} \mathrm{~b}$. Purified eosinophil and neutrophil granule proteins were kindly provided by Dr Inge Olsson, Lund, Sweden (see Venge and Olsson, 1980). The $\mathrm{O}_{2}$ uptake of isolated rat heart cells was measured with an $\mathrm{O}_{2}$ electrode, and the integrity of the mitochondrial respiratory chain was assessed using isolated rat heart cell mitochondria (Tai et al., 1982).

The concentration of eosinophil granule basic proteins in serum was assayed using a mouse monoclonal antibody (EG2) which detected a common epitope on eosinophil cationic protein (ECP) and eosinophil neurotoxin (EPX). These two proteins were assayed together in serum, using this monoclonal antibody, in a competitive inhibition enzyme immunoassay with peroxidase-linked rabbit antimouse immunoglobulin (Tai and Spry, unpublished).

\section{Results}

\section{Blood eosinophil counts}

All 11 patients with eosinophilic endomyocardial disease presented with hypereosinophilia (blood eosinophil counts more than $1.5 \times 10^{\circ} /$ litre), but 3 patients had normal blood eosinophil counts fe्ष several weeks or months during their illness. In or: patient, the eosinophil count became normal spontá: neously. In 2 other patients, normal counts developed after steroid treatment had begun, and in one of them, the eosinophil count has remained normal for 18 months, except for short periods when it ros above $1 \times 10^{9} /$ litre.

\section{Degranulated blood eosinophils}

Twelve of the 15 patients with the hypereosino philic syndrome had more than $0.5 \times 10^{\circ} /$ lit $\overrightarrow{\mathrm{ES}}$ degranulated blood eosinophils. Eleven of these patients were shown to have eosinophilic endomy cardial disease. One patient with endomyocardia disease had only intermittently raised blood eosine phil counts and few degranulated blood eosinophils Another patient, who had many degranulated bloog eosinophils at the start of his illness, has subsequenti been found to have a normal heart, judged clinically and by echocardiography, and degranulated celts have disappeared from his circulation. No degranurlated blood eosinophils have been seen in blood film from the 2 other patients with hypereosinophilif without endomyocardial disease.

One patient showed a remarkable associago $\overrightarrow{8}$ between a rise in the number of degranulated bfood eosinophils and the development of acute endong cardial disease. He had had blood eosinophil count of between 5 and $10 \times 10^{\circ} /$ litre for 2.5 years with $n$ evidence of endomyocardial disease. Then he becampin very ill with fever, exhaustion, cough, abdomin pain and rapid weight loss. The eosinophil count ros to more than $100 \times 10^{9} /$ litre, and for the first time many of them were found to be degranulated. The heart enlarged and cardiological investigations now showed that he had developed bi-ventricular endomyocardial disease in the acute necrotic stage. was treated with steroids which produced a rap improvement in systemic and cardiological symp. toms and signs. Several months later he was able tio return to work, and echocardiography and endomyor cardial biopsy have shown that his disease has nof progressed from the acute necrotic stage.

The mean concentrations of serum ECP and EP together, in 13 patients with hypereosinophilic syndrome without heart disease was $2030 \pm 2900 \mathrm{ng} / \mathrm{n}$ (mean \pm s.d.). The mean level in 9 patients witho eosinophilic endomyocardial disease was signifivo cantly higher: $4540 \pm 2600 \mathrm{ng} / \mathrm{ml}$.

\section{Injury to rat heart cells in vitro}

Supernatants from eosinophils which had been stimulated with $\mathrm{C} 3 \mathrm{~b}$ gave rise to both a dose dependent cytotoxic effect and a stimulation of $\omega_{1}$ 
uptake by isolated rat heart cells in vitro. This effect on respiration was blocked by ouabain, showing that it occurred as a result of an increase in the activity of $\mathrm{Na}^{+} / \mathrm{K}^{+}$ATPase in the plasma membrane. These eosinophil supernatants also inhibited pyruvate dehydrogenase and 2-oxoglutarate dehydrogenase in isolated rat heart mitochondria. Neutrophil secretion products did not have these effects. Preliminary studies with purified ECP, and a mouse monoclonal antibody which binds to ECP and EPX, have suggested that ECP was responsible for the cytotoxicity in eosinophil supernatants. Single cell preparations from other tissues were not injured by eosinophil supernatants, and equal concentration of other unrelated proteins, with a similar basic charge, had no effect on heart cell viability or respiration or isolated mitochondrial enzymes (Tai et al., 1982).

\section{Discussion}

This study was designed to review the evidence that eosinophils could damage the heart and give rise to endomyocardial disease. Three separate lines of evidence support this view. The first is derived from clinical studies on patients with hypereosinophilia. The second is related to the detection of degranulated eosinophils in the blood and tissues, and the third is the direct demonstration that eosinophil granule proteins can injure isolated heart cells in vitro.

\section{Clinical studies}

These have provided some of the most compelling evidence that eosinophils may cause endomyocardial disease. Two reports have listed the wide range of clinical disorders in which hypereosinophilia may be associated with endomyocardial disease (Olsen and Spry, 1979; Schooley et al., 1980). The most likely common factor in these diseases was the presence of a marked blood eosinophilia which, in many patients, clearly preceded the onset of clinical evidence of heart disease. The temporal relationship between eosinophilia and heart disease was clearly shown in 6 of the 11 patients with endomyocardial disease reported here. It is interesting to consider why some patients present both with heart disease and hypereosinophilia, and why others only develop heart disease later. The most likely explanation is that this type of heart disease is asymptomatic in its early stages unless there is a widespread and severe carditis. For this reason most patients will only present with early heart involvement if they have other complications of the underlying disease which is producing the eosinophilia. This may explain why it has been impossible as yet to detect and define the early stages of tropical endomyocardial fibrosis. Patients who develop a moderate eosinophilia in areas of endemic parasitic disease, including filariasis, may not be distinguishable from other parasitized individuals until their heart disease is in a late stage, when blood eosinophil counts may have returned to normal. The disappearance of high blood eosinophil counts has been documented in patients with eosinophilic endomyocardial disease, and these patients appear to be an intermediate group between patients with the tropical and eosinophilic forms of endomyocardial disease.

\section{Degranulated blood eosinophils}

The presence of degranulated blood eosinophils in patients with hypereosinophilia and endocardial thrombi and fibrosis has been known for over 60 years (Shapiro, 1919). It was suggested (Spry and Tai, 1976) that these cells had released their granule contents which damage the endomyocardium leading to the development of endomyocardial disease. This hypothesis has been supported by studies on the patients reported here. Ten patients with eosinophilic endomyocardial disease had many degranulated blood eosinophils in their circulation. Unfortunately, in one patient with acute necrotic cardiac lesions, eosinophils were not available for study. In one other patient, although degranulated cells were found for 3 months, there was no clinical evidence of endomyocardial disease. As degranulated cells then disappeared from the blood, invasive cardiac studies (which might have shown early lesions) were not carried out. The clinical significance of the presence of degranulated eosinophils in blood smears was confirmed by Jaski et al., (1978) who noted the presence of these cells during life in a patient with carcinoma of the lung-induced hypereosinophilia. who was shown to have endomyocardial lesions at post-mortem. We have studied a similar patient, and documented an identical sequence of events.

The recent demonstration of degranulated eosinophils in both the eosinophilic and tropical forms of endomyocardial disease (Olsen, 1983) raises the possibility that degranulation may occur locally in the heart, and degranulated eosinophils may not be present in the circulation of these patients. This important finding strengthens the view that both forms of the disease have a common pathogenesis related to the presence of eosinophil granule basic proteins in the heart. This view will be strengthened if it can be shown that these toxic proteins are present in endomyocardial biopsies from patients in early stages of both forms of the disease.

Raised levels of eosinophil granule basic proteins were found in the circulation of patients who had many degranulated blood eosinophils. The significance of this finding is not clear as patients with an eosinophilia (which was the result of parasitic diseases) and who did not have endomyocardial disease 
have been found to have equally high blood eosinophil granule basic protein levels (Tai, unpublished). It is possible that circulating eosinophil granule basic proteins have an initial toxic effect on the heart, causing eosinophils to localize there and degranulate. Alternatively, a small proportion of patients who develop hypereosinophilia may lack effective inhibitors for the potentially cardiotoxic effects of eosinophil granule basic proteins. This latter explanation could account for the low incidence of endomyocardial disease in patients with asthma-induced hypereosinophilia (Olsen and Spry, 1979).

\section{Heart cell injury in vitro}

Human eosinophil granule basic proteins have been shown to injure rat heart cells in vitro, and inhibit isolated mitochondrial respiration. Less than $10^{-6} \mathrm{~mol} /$ litre eosinophil granule basic proteins produced these effects, and these concentrations are likely to be achieved within the heart in vivo. Due to their strong charge, eosinophil granule basic proteins are likely to bind closely to cell membranes. This may be the first step in membrane damage which alters permeability to sodium. It is not known whether these basic proteins enter cardiac cells, but if they do so, they are capable of inhibiting mitochondrial respiration directly. Either (or both) of these events are likely to lead to cardiac cell death.

Previous studies, which attempted to show a toxic effect of eosinophils on cardiac cells, met with little success (Epps and Bankhurst, 1978; Parrillo and Fauci, 1978). ${ }^{51} \mathrm{Cr}$ labelled target cells, including cultured atrial cells, were treated with antibody and/or $\mathrm{C} 3 \mathrm{~b}$ and incubated with blood eosinophils. Only a small proportion of the chromium was released. However, neither study measured the concentration of eosinophil granule proteins which were released in these experiments, and this may have been small. The results do, however, suggest that antiheart antibody, or direct complement activation on the surface of cardiac cells, are unlikely to be involved in the induction of eosinophil dependent endomyocardial damage. This is supported by studies on patients' sera which have failed to detect antiheart antibody (Davies, unpublished). However, it should be noted that blood eosinophils which have little initial cytotoxic capacity in normal people may be activated in vitro, and eosinophils in patients with an eosinophilia are metabolically and functionally more effective than normal. These changes are linked to alterations in oxygen metabolism (Pincus et al., 1981), and it is possible that the production of oxygen radicals augments the cytotoxic effects of the eosinophil granule basic proteins (Olsen and Spry, 1979).

Two important questions remain to be answered: Why is the endocardium so susceptible to eosinophil dependent injury, and what determines the rate which endocardial disease progresses to the la fibrotic stage? Selective injury to the endocardium may be related to regional differences in cardia blood supply, metabolism or capacity to repalf injury. Alternatively, toxic eosinophil granule base proteins may be concentrated in the endocardiur? Each of these possibilities can now be studied, as $\rightarrow$ model of eosinophil granule basic protein dependem cardiac injury and methods to do so are bein developed in rats (Davies et al., unpublished).

The second question is more difficult to approach but it is of considerable importance as it is now likety that tropical endomyocardial disease is a similar, ba more slowly progressing, form of eosinophilic end\&. myocardial disease (Davies et al., 1983b). The late. fibrotic lesions in both forms of the disease af identical (Brockington and Olsen, 1973), and it has been thought for many years that they might have common pathogenesis related, in some way, to the presence of eosinophils in blood or tissues (Olsen and Spry, 1979). Unfortunately, it is impossible to studf mechanisms of chronic injury using isolated heaft cells in vitro, but it may also be possible to approa電 this question using the rat model of eosinoph dependent injury. Although these issues, and othet questions, such as the geographical distributiob $\overrightarrow{0}$ tropical endomyocardial fibrosis, remain to beres solved, studies on the interaction between eosinopits and heart muscle have provided many new proaches to the study of both eosinophilic an tropical endomyocardial disease. It is hoped that thas will provide further insights into the mechanisms of this disease and will lead to effective methods for prevention, early diagnosis and treatment.

\section{Acknowledgments}

We are grateful to the British Heart Foundation and Wellco Trust for supporting this work, and to our many clinical a rd scientific colleagues who have referred patients. and contributed 95 the ideas and results reviewed here. This work is part of a mufr. centre research project on endomyocardial disease organized by tipe International Society and Federation of Cardiology.

\section{References}

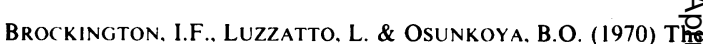
heart in eosinophilic leukaemia. African Journal of Mediga Science, 1, 343.

BRockington. I.F. \& OlSEN, E.G.J. (1973) Löffler's endocardikis and Davies' endomyocardial fibrosis. American Heart Journal, 308.

Davies. J., SPRy, C.J.F., Sapsford, R., Olsen, E.G.J., DuPere G., OAKLeY, C.M. \& Goodwin, J.F. (1983a) Cardiovascular features of eleven patients with eosinophilic endomyocardfil disease. Quarterly Journal of Medicine, 52, 23.

Davies, J., Spry, C.J.F., Vijayaraghavar, G. \& de Souza, J.A (1983b) A comparison of the clinical and cardiological features of endomyocardial disease in temperate and tropical regions. $P o$ 尺ुgraduate Medical Journal, 59, 179. 
EPPS, D.E. VAN \& BANKHURST, A.D. (1978) Human eosinophils: surface receptors and antibody-dependent cytotoxicity. Journal of Laboratory and Clinical Medicine, 91, 612.

JASKI, B.E., GoetZl, E.J., SAID, J.W. \& Fishbein, M.C. (1978) Endomyocardial disease and eosinophilia. Report of a case. Circulation, 57, 824.

LÖFFLER, W. (1936) Endocarditis parietalis fibroplastica mit Bluteosinophilie; ein eigenantigen Krankheitsbild. Schweizerische medizinische Wochenschrift, 17, 817

OLSEN, E.G.J. (1983) Pathological aspects of endomyocardial disease. Postgraduate Medical Journal, 59, 135.

OLSEN, E.G.J. \& SPRY, C.J.F. (1979) The pathogenesis of Löffler's endomyocardial disease and its relationship to endomyocardial fibrosis. Progress in Cardiology, 8, 281.

Parrillo, J.E., Borer, J.S., Henry. W.C. WolfF. S.M. \& Fauci. A.S. (1979) The cardiovascular manifestations of the hypereosinophilic syndrome: prospective study of 26 patients, with review of the literature. American Journal of Medicine, 67, 572.

Parrillo, J.E. \& Faucl, A.S. (1978) Human eosinophils. Purification and cytotoxic capacity of eosinophils from patients with the hypereosinophilic syndrome. Blood, 51, 457

Pincus, S.H., SChooley, W.R.. Dinapoli. A.M. \& Broder, S. (1981) Metabolic heterogeneity of eosinophils from normal and hypereosinophilic patients. Blood, 58, 1175.

SCHOOLEY, R.T., PARRILlo, J.E., WolfF, S.M. \& FauCi, A.S. (1980)
Management of the idiopathic hypereosinophilic syndrome. In The Eosinophil in Health and Disease. (eds. Mahmoud. A.A.F. and Austen K.F.), p. 323. Grune and Stratton, New York.

SHAPIRO, L.G. (1919) Eosinophilic (polymorphonuclear) leukemia Proceedings of the New York Pathological Society, 19,73.

SPRY, C.J.F. (1978) Eosinophils as effector cells in disease. Schweizerische medizinische Wochenschrift, 108, 1572.

SPRY, C.J.F. (1982) The hypereosinophilic syndrome: clinical features. laboratory findings and treatment. Allergy, 37, 539.

SPRY, C.J.F., Davies, J.. Tai, P.C., Olsen, E.G.J., OAKLEY, C.M. \& GoodwIN, J.F. (1982) Clinical features of fifteen patients with the hypereosinophilic syndrome. Quarterly Journal of Medicine, 52, I

SPRY, C.J.F. \& TAI, P.C. (1976) Studies on blood eosinophils II Patients with Löffler's cardiomyopathy. Clinical and Experimental Immunology, 24, 423.

Tai, P.C.. Hayes, D.J., Clark, J.B. \& SPRy. C.J.F. (1982) Toxic effects of human eosinophil secretion products on isolated rat heart cells in vitro. Biochemical Journal. 204, 75.

VENGE, P. \& OLSSON, I. (1980) The role of human eosinophils in the inflammatory reaction. Allergy, 35, 15.

Yam. L.T.. LI. C.Y., Necheles, T.F. \& Katayama. I. (1972) Pseudoeosinophilia. eosinophilic endocarditis, and eosinophilic leukemia. American Journal of Medicine, 53, 193.

ZUCKER-FRANKLIN. D. (1971) Eosinophil function and disorders Advances in Internal Medicine, 19, 1. 